\title{
ABO blood group, secretor state, and susceptibility to recurrent urinary tract infection in women
}

\author{
D F KINANE, C CAROLINE BLACKWELL, R P BRETTLE, D M WEIR, F P WINSTANLEY, \\ R A ELTON
}

\begin{abstract}
ABO blood group and secretor state was determined in 319 women with recurrent urinary tract infection and compared with those of a control group of 334 women of similar age ranges. Women of blood groups $B$ and $A B$ who are non-secretors of blood group substances showed a significant relative risk of recurrent urinary tract infection of $3.12(95 \%$ confidence limits, 1.49 and 6.52$)$ in comparison with other types. This appears to be a genuine example of synergy in which absence of anti-B isohaemagglutinin and secretor substances combines to give an increased risk of recurrent urinary tract infection.

Determination of blood group and secretor state may provide additional information in identifying those at risk.
\end{abstract}

\section{Introduction}

Correlation between $\mathrm{ABO}$ blood group and susceptibility to certain infectious diseases is well documented. ${ }^{1-6}$ In studies of a Chilean population, patients of blood group B had 50\% greater probability than those of other blood groups of contracting Escherichia coli urinary tract infections. ${ }^{4}$

The antigens of $\mathrm{ABO}$ blood groups are present in two forms:

\footnotetext{
Department of Bacteriology, University of Edinburgh Medical Scl o , 'dinburgh EF 8 9AG

D F KINANE, BDs, research fellow

C CAROLINE BLACKWELL, PHD, lecturer

D M WEIR, MD, FRCP, reader

F $P$ WINSTANLEY, BSC, research associate

Department of Infectious Diseases, City Hospital, Edinburgh EH10 5SB

R P BRETTLE, MRCP, senior registrar
}

Medical Computing and Statistics Unit, University of Edinburgh Medical School, Edinburgh EH8 9AG

R A ELTON, PHD, lecturer (a) alcohol-soluble in tissues of all subjects, and (b) water-soluble in most body fluids and organs of secretors. The non-secretor state has been linked with the susceptibility to certain infectious agents. ${ }^{7-9}$ Haverkorn and Goslings ${ }^{7}$ found that patients in blood groups other than $\mathrm{O}$ and non-secretor patients were most susceptible to rheumatic fever and rheumatic heart disease, and carriage of group A streptococci was observed more often in non-secretors.

We analysed the distribution of $\mathrm{ABO}$ blood groups and secretor state among women with recurrent urinary tract infections in Edinburgh.

\section{Subjects}

Regular attenders at the pyelonephritis clinic of the City Hospital, Edinburgh, were asked to provide a specimen of blood and saliva for determination of blood group and secretor state. All patients in this survey were girls and women aged 10-80 (mean 49) years. None had predisposing factors such as stones.

The patients were divided prospectively into two categories:

Patients with pyelonephritis-Those with unequivocal radiological evidence of chronic pyelonephritis-that is, focal loss of parenchyma associated with distortion of calyces-or those with a history and clinical and laboratory findings consistent with acute pyelonephritis.

Patients with uncomplicated urinary tract infections-Those patients with normal appearance on intravenous pyelograms, but with appreciable bacteriuria $\left(10^{5}\right.$ organisms $\left./ \mathrm{ml}\right)$ and a history of recurrent urinary tract infections.

Controls-Control data were produced by testing 334 samples of plasma from female donors (age range 18-60) from the survey area for the presence of secretor substances. Further control data for distribution of blood groups were available for 6662 donors from the same area as that from which our patients were drawn. ${ }^{10}$

\section{Methods}

Blood from each patient was collected at the clinic and stored in heparinised tubes. The saliva from each patient was boiled for $20 \mathrm{~min}$, centrifuged at $5000 \times g$ for $10 \mathrm{~min}$, and the supernate stored briefly at $4^{\circ} \mathrm{C}$ until tested for the presence of blood group antigen. Blood group was determined by agglutination tests in plastic wells. Secretor state was determined as described by Mollinson ${ }^{11}$ with saliva or 
plasma samples. Saliva or plasma from secretors and non-secretors representative of the four blood groups were used as controls for each experiment. Agglutinins used were anti-A and anti-B sera and Ulex europaeus lectin. The red blood cells used were $A_{2}, B$, and $O$.

Linear logistic multiple regression ${ }^{12}$ was used to compare the $\mathrm{ABO}$ blood group and secretor state categories of the study and control groups. This also gave estimates and confidence limits for relative risk of urinary tract infection between categories. $\chi^{2}$ tests were used for analysis of two-way frequency tables.

\section{Results}

No significant differences in the proportion of secretors or the distribution of $\mathrm{ABO}$ blood groups were found between the two diagnostic categories of patients with urinary tract infections; all 319 patients were considered as one group for comparisons with controls. The table shows the distribution of different blood groups and secretor state for the study group and for the smaller series of controls in whom secretor status had been determined. For the study group, the table shows also the ratios of observed prevalences to those expected from the pattern found in controls.

Distribution of blood groups in study group and in two groups of controls, those in whom secretor state was determined (group 1) and those in whom it was unknown (group 2) (numbers in parentheses are ratios of distribution found in study group to that expected from control data)

\begin{tabular}{lcccc}
\hline & \multicolumn{5}{c}{ Blood group } \\
\cline { 2 - 5 } & $\mathrm{O}$ & $\mathrm{A}$ & $\mathrm{B}$ & $\mathrm{AB}$ \\
\hline Control group 1 $(\mathrm{n}=334):$ & & & & \\
$\quad$ Secretors & 124 & 74 & 35 & 12 \\
$\quad$ Non-secretors & 49 & 30 & 7 & 3 \\
Study group ( $=319):$ & $110(0.93)$ & $63(0.88)$ & $32(0.96)$ & $7(0.64)$ \\
$\quad$ Secretors & $52(1.11)$ & $28(0.98)$ & $18(2 \cdot 69)$ & $10(3.49)$ \\
$\quad$ Non-secretors & 3323 & 2410 & 715 & 214 \\
Control group 2 ( $\mathrm{n}=6662)$ & & & & \\
\hline
\end{tabular}

To test the dependence of risk of urinary tract infection on ABO blood group or secretor state, a linear logistic multiple regression was carried out on the two samples. This examines the influence of the three main effects (anti-A, anti-B, and secretor state) and their interactions on the proportion of subjects with urinary tract infection, using approximate $\chi^{2}$ tests. Presence or absence of anti-A was found not to have a significant effect, but there was a significant interaction between the anti-B and secretor effects; subjects lacking both anti-B and secretor substance - that is, those of blood groups B and AB who are non-secretors-had a higher risk of recurrent urinary tract infection, whereas subjects lacking only one of these two did not differ significantly in risk from those with both factors present. Thus the data fit extremely well $\left(\chi^{2}=1 \cdot 86,6 \mathrm{df}\right)$ to a model in which the non-secretor in blood groups $\mathrm{B}$ and $\mathrm{AB}$ have a highly significant excess risk $\left(\chi^{2}=9 \cdot 12,1 \mathrm{df}, \mathrm{p}<0.01\right)$ over all other types. The relative risk of urinary tract infection for these subjects as compared with other types was estimated as $3 \cdot 12$, with $95 \%$ confidence limits of 1.49 and 6.52. This appears to be a genuine example of synergy with absence of both anti-B and secretor substance being necessary to give an increased risk of urinary tract infection. The ratios of observed to expected prevalence in the table show this finding clearly, with only those for non-secretors in blood groups $\mathrm{B}$ and $\mathrm{AB}$ differing substantially from unity.

The larger series of controls (in whom only $\mathrm{ABO}$ group and not secretor state was known) did not differ significantly from the smaller series in their blond group frequencies, but showed a highly significant difference $\left(\chi^{2}=16.34,3 \mathrm{df}, \mathrm{p}<0.001\right)$ from the patients wit 2 urinary tract infections (table) (14\% in blood groups $\mathrm{B}$ and $\mathrm{AB} v 21 \%$ ). This confirms previous findings and is consistent with the suggested model in which non-secretors in blood groups $B$ and $A B$ have an increased likelihood of recurrent urinary tract infection. The proportion of anti-B negative patients in the study group is inflated by the excess prevalence of those who are also non-secretors, even though anti-B negative secretors are not at a higher risk. Thus, absence of anti-B when considered alone shows a significant risk relative to presence of anti-B ( $95 \%$ confidence limits of 1.24 and 21.7 (table)). This risk is lower than the threefold increase in risk found for non-secretors in blood groups B and AB, because the anti-B negative group is "diluted" with anti-B negative secretors.

\section{Discussion}

The highly significant excess of non-secretors in blood groups $B$ or $A B$ in the study group strongly suggests a synergistic link between the absence of anti-B isohaemagglutinin and secretor substances and an increased risk of recurrent urinary tract infection in women. In the controls the prevalence of nonsecretors of $26.6 \%$ reflects an increased incidence of nonsecretors found in Scottish and Irish ethnic groups compared with that reported for other regions in the United Kingdom. ${ }^{13}$ This highlights the need to use local control groups in surveys of this nature.

The association of infections with $\mathrm{ABO}$ blood group and secretor state ${ }^{1-9}$ has led to investigation of the mechanisms concerned. ${ }^{61415}$ Many bacteria cross-react with ABO blood groups. ${ }^{14-20}$ Drach et $a l^{18}$ found A and B blood group activity in $47 \%$ of 34 urinary tract pathogens. Isohaemagglutinins may have an important protective role against these cross-reacting bacteria. ${ }^{1519}$ Our finding that individuals of blood groups $B$ and $A B$ with no anti-B isohaemagglutinins are more susceptible to urinary tract infection is interesting in that Drach ${ }^{18}{ }^{20}$ found antigens like those of blood groups $A$ and $B$ on urinary tract pathogens, often with both group $A$ and group $B$ activity on the same bacterium. These workers noted that anti-B titres were sometimes raised in the disease but anti-A ones were not, suggesting that the antigen to blood group A was less immunogenic on the bacteria or that there were deficiencies in recognition of antigens of blood group A. Springer et al ${ }^{16}$ found $E$ coli 086 B:7 to be highly interactive with the blood group: thus growth was retarded in the presence of anti-B sera; if complement was added a bactericidal reaction ensued. Isohaemagglutinins may also interact with blood group like antigens on bacterial cell walls to inhibit attachment to uroepithelial or periurethral cells. Thus persons capable of producing anti-Bisohaemagglutinins may have a greater degree of protection against urinary tract infection. In general, the extent of bacterial cross-reaction with $\mathrm{ABO}$ blood groups suggests that a selective advantage linked to ABO blood group might operate in combating infections.

Secretor state is associated with certain differences in immunoglobulin concentrations in that anti-B serum IgA and (in white people) serum IgG concentrations are lower in non-secretors than in secretors. ${ }^{21}{ }^{22}$ This suggests that non-secretors may have less effective immune protection than secretors and is consistent with our findings that non-secretors were more susceptible to recurrent urinary tract infection. This probably entails the participation of other factors, and the finding that excretion of blood group substances in urine is an active process in the kidney and not a simple filtration proces $\mathrm{s}^{23}$ suggests that these substances may have a protective part to play in secretions. Boat et $a l^{24}$ found that $\mathrm{ABH}$ blood group substances of saliva inhibited haemagglutination of influenza $B$ virus and suggested that some of these substances may interfere with access of influenza virus to binding sites. One of the protective roles of these blood group substances may be their ability to occupy or in some way interfere with binding sites either on the bacterium or on the epithelial cell with possible effects on bacterial colonisation and subsequent invasion and infection; this appeared to be the case in our studies on diabetic mice. ${ }^{25}$

Determination of blood groups and secretor state may provide additional information in identifying individuals who are at risk. In children under the age of 5 the combination of infection and vesicoureteral reflux predisposes to renal scarring. ${ }^{26}$ Few if any tests are available for providing prognostic information for adults with a normal urinary tract and a history of recurrent infection. Blood group and secretor state may be of relatively minor importance in relation to all other aspects of susceptibility. Nevertheless, it may be worthwhile for the clinician to take account of $\mathrm{ABO}$ and secretor state in addition to already established factors in the assessment of patients with urinary tract infection when considering their long-term susceptibility and management. Meanwhile, there is a good case for research workers concerned with mechanisms of microbial pathogenicity 
to add this consideration to an extending list of host factors that bear on host-parasite interactions.

This work was supported by grant $\mathrm{K} / \mathrm{MRS} / 50 / \mathrm{C} 22$ from the Biomedical Research Committee, Scottish Home and Health Department and by grants G81/103811/SB and G979/74/S from the Medical Research Council.

We thank the Blood Transfusion Service, Royal Infirmary, Edinburgh for supplying reagents for blood grouping and determination of secretor state and for undertaking random verification checks on selected saliva (50) and plasma (48) samples; Dr J McMurdoch and Dr J A Gray for permission to study their patients; the doctors and staff of Ward 14, City Hospital, Edinburgh; and Professor J G Collee and Messrs A McGowan and G Neill for their help and advice.

\section{References}

1 Barua D, Paguio AS. ABO blood group and cholera. Ann Hum Biol 1977; 4:489-92.

${ }^{2}$ Socha W; Bilinska M, Kaczera Z, Padjak E, Stankiewicz D. Escherichia coli and ABO blood groups. Folia Biol (Krakow) 1969;17:259-69.

${ }^{3}$ Robinson MG, Tolchrin D, Halpern C. Enteric bacterial agents and the ABO blood groups. Am $\mathcal{F}$ Hum Genet $1971 ; 23: 135-45$.

${ }^{4}$ Cruz-Coke R, Parades L, Montengro A. Blood groups and urinary microorganisms. F Med Genet 1965;2:185-8.

${ }^{5}$ Foster MT, Labrum AH. Relation of infection with Neisseria gonorrhoeae to blood groups. $\mathcal{F}$ Infect Dis 1976;133:329-30.

6 Miler JJ, Novotny P, Walker PD, Harris JRW, MacLennan IPB. Neisseria gonorrhoeae and ABO isohaemagglutinins. Infect Immun 1977; $15: 713-9$.

${ }^{7}$ Haverkorn MJ, Goslings WRO. Streptococci ABO blood groups and secretor status. Am $\mathcal{F}$ Hum Genet 1969;21:360-75.

${ }^{8}$ Pradhan AC, Chawla TN, Samuel KC, Pradhan S. The relationship between periodontal disease and blood groups and secretor status. $\mathcal{f}$ Periodont Res 1971;6:194-300.

${ }^{9}$ Chaudhuri A, Das Adchickary CR. Possible role of blood group secretory substances in the aetiology of cholera. Trans $R$ Soc Trop Med Hyg $1978 ; 72: 664-5$.
${ }^{10}$ Kopeć AC. The distribution of the blood groups in the United Kingdom. Oxford: Oxford University Press, 1970:26.

11 Mollinson PL. Blood transfusion in clinical medicine. 6th ed. Oxford: Blackwell, 1979:414-82.

12 Armitage P. Statistical methods in medical research. Oxford: Blackwell, $1971: 375$

${ }^{13}$ Lincoln PJ, Dodd BE. Variation in secretor and Lewis type frequencies within the British Isles. $\mathcal{F}$ Med Genet 1972;9:43-5.

14 Springer GF, Williamson P, Brandes WC. Blood group activity of Gramnegative bacteria. F Exp Med 1961;113:1177-93.

${ }^{15}$ Muschel L, Osawa E. Human blood group substances B and Escherichia coli 086. Proc Soc Exp Biol Med 1959;101:614-7.

${ }^{16}$ Springer GF. Importance of blood group substances in interactions between man and microbes. Ann NY Acad Sci 1970;169:134-52.

17 Springer GF. Blood group and Forssman antigenic determinants shared between microbes and mammalian cells. Prog Allergy 1971;15:9-77.

18 Drach GW, Reed WP, Williams RC. Antigens common to human and bacterial cells : urinary tract pathogens. F Lab Clin Med 1971 ;78:725-35.

19 Check JH, O'Neill EA, O'Neill KE, Fuscaldo KE. Effect of anti-B antiserum on the phagocytosis of Escherichia coli. Infect Immun 1972;6: 95-6.

${ }^{20}$ Drach GW. Antigens common to human and bacterial cells: tolerance or resistance to urinary and surgical infections. Surg Forum 1971 ;22:67-8.

${ }^{21}$ Grundbacher FJ, Shreffler DC. Effects of secretor, blood and serum groups on isoantibody and immunoglobulin levels. Am F Hum Genet $1970 ; 22: 194-202$.

22 Grundbacher FJ. Immunoglobulins, secretor status and the incidence of rheumatic fever and rheumatic heart diseases. Hum Hered 1972;25: 399-404.

${ }^{23}$ Kalinowski $M$. Urinary elimination of group substances $\mathrm{ABO}(\mathrm{H})$ in different renal diseases. Folia Haematol (Liepz) 1972;100:144-63.

${ }^{24}$ Boat TF, Davis J, Stern RC, Cheng PW. Effect of blood group determinants on binding of human salivary mucous glycoproteins to influenza virus. Biochim Biophys Acta 1978;540:127-33.

${ }^{25}$ Weir DM, Blackwell CC, McLean CA. Impaired bacterial binding to peritoneal exudate cells from mice with alloxan induced diabetes. fournal of Clinical and Laboratory Immunology 1981 ;5:37-40.

${ }^{26}$ Rolleston GL, Shannon FT, Utley JF. Relationship of infantile cytoureteric reflux to renal damage. $\mathrm{Br} \mathrm{Med} \mathcal{F} 1970 ; \mathrm{i}: 460-3$.

(Accepted 19 April 1982)

\title{
Severe hypoglycaemia due to combined use of parenteral nutrition and renal dialysis
}

\author{
J D B MILLER, J BROOM, G SMITH
}

\begin{abstract}
A 24-year-old woman who had sustained serious injuries in a road traffic accident required renal dialysis daily and was fed intravenously with a solution containing $25 \%$ dextrose. Subsequently insulin had to be added to the parenteral fluid to maintain blood glucose concentrations at physiological values. On one occasion parenteral feeding was continued until dialysis was started; she became comatose and the plasma glucose concentration was found to be $<1 \mathrm{mmol} / 1(18 \mathrm{mg} / 100 \mathrm{ml})$. She responded rapidly to a $50 \mathrm{ml}$ intravenous bolus of $50 \%$ dextrose.

When parenteral feeding and dialysis are used simultaneously glucose passes across the semi-permeable membrane from the blood to the dialysate so that hypoglycaemia may occur. Insulin added to the parenteral fluid further decreases blood glucose concentrations. Stopping parenteral feeding 30-45 minutes before dialysis is started eliminates this danger of hypoglycaemia.
\end{abstract}

Department of Surgery, University of Aberdeen, Aberdeen AB9 2ZD

J D B MILLER, CHM, FRCs, senior lecturer in surgery

J BROOM, MB, CHB, lecturer in surgery and chemical pathology

G SMITH, DSC, MD, regius professor of surgery

\section{Introduction}

Parenteral nutrition and renal dialysis are sometimes used together to treat severely ill patients. These supportive techniques may individually cause fluid, electrolyte, and metabolic disturbances; we propose that in the case described below their combined use caused severe and unexpected hypoglycaemia.

\section{Case report}

A 24-year-old woman was injured in a road traffic accident and sustained a traumatic pneumothorax, a fractured pelvis, spinal damage with paraplegia, a ruptured and in parts devitalised small intestine, and a ruptured thoracic aorta. She survived aortic repair with a Dacron graft, small-bowel resection, and drainage of the pneumothorax. Her postoperative course was complicated by ventilatory difficulties, septicaemia, and acute renal failure. From the third day after admission she required daily renal dialysis for three weeks. During this time she was fed intravenously with a solution containing $25 \%$ dextrose and $\mathbf{4} \cdot 25 \%$ amino-acids delivered via a subclavian vein. Ten days after admission she underwent a further operation for drainage of an empyema. The stress associated with the initial injury, and subsequent septic complications, required that soluble insulin in doses of 15-25 U/1 be added to the parenteral nutrition fluid to maintain the blood glucose concentration at physiological values. A total of 2.0-2.5 1 parenteral feed was delivered daily over 18 hours 